\title{
REDUCING AND TOROIDAL DEHN FILLINGS ON 3-MANIFOLDS BOUNDED BY TWO TORI
}

\author{
SANGYOP LEE
}

\begin{abstract}
We show that if $M$ is a simple 3-manifold bounded by two tori such that $M\left(r_{1}\right)$ is reducible and $M\left(r_{2}\right)$ is toroidal, then $\Delta\left(r_{1}, r_{2}\right) \leq 2$, answering a question raised by Gordon. To do this, we first prove that there exists only one simple 3-manifold having two Dehn fillings of distance 3 apart one of which yields a reducible manifold and the other yields a 3-manifold containing a Klein bottle.
\end{abstract}

\section{Introduction}

Let $M$ be a compact connected orientable 3-manifold with a torus boundary component $\partial_{0} M$ and $r$ a slope, the isotopy class of an essential simple closed curve, on $\partial_{0} M$. The manifold obtained by $r$-Dehn filling is defined to be $M(r)=$ $M \cup J$, where $J$ is a solid torus glued to $M$ along $\partial_{0} M$ so that $r$ bounds a disk in $J$.

Following [22], we say that $M$ is simple if it contains no essential sphere, torus, disk or annulus. For two slopes $r_{1}$ and $r_{2}$ on $\partial_{0} M$, the distance $\Delta\left(r_{1}, r_{2}\right)$ denotes their minimal geometric intersection number. For simple manifolds $M$, if both $M\left(r_{1}\right)$ and $M\left(r_{2}\right)$ fail to be simple, then the upper bounds for $\Delta\left(r_{1}, r_{2}\right)$ have been established in various cases. See [8] for more details.

For example, Oh [18] and independently $\mathrm{Wu}$ [23] showed that for a simple manifold $M$, if $M\left(r_{1}\right)$ is reducible and $M\left(r_{2}\right)$ is toroidal then $\Delta\left(r_{1}, r_{2}\right) \leq 3$. Furthermore, $\mathrm{Wu}[22]$ also showed that if one puts an additional condition $H_{2}\left(M, \partial M-\partial_{0} M\right) \neq 0$, then $\Delta\left(r_{1}, r_{2}\right) \leq 1$. In particular, this homological condition holds if $M$ has a boundary component with genus greater than one or if $M$ has more than two boundary tori. Note that $M$ has no boundary sphere, for $M$ is simple. It is natural then to consider the following question raised by Gordon [8, Question 5.1]; if $\partial M$ consists of two tori, is it possible that $\Delta\left(r_{1}, r_{2}\right)=3$ ? In this paper we give a negative answer to the question.

Theorem 1.1. Let $M$ be a simple 3-manifold with boundary a union of two tori. If $r_{1}$ and $r_{2}$ are slopes on one boundary component $\partial_{0} M$ such that $M\left(r_{1}\right)$ is reducible and $M\left(r_{2}\right)$ is toroidal, then $\Delta\left(r_{1}, r_{2}\right) \leq 2$.

Received by the editors February 21, 2005.

2000 Mathematics Subject Classification. 57N10.

Key words and phrases. Reducible manifolds, Toroidal manifolds, Dehn fillings. 
Our theorem is sharp because Eudave-Muñoz and Wu [5, Theorem 2.6] have given infinitely many simple manifolds which are bounded by two tori and admit reducing and toroidal Dehn fillings at distance 2 .

Oh [19] showed that if one Dehn filling yields a reducible manifold and another yields a manifold containing a Klein bottle, then the distance between their filling slopes is not greater than 3. On the other hand, Boyer and Zhang [1, p.286] gave an example of a simple manifold showing Oh's result is sharp. This simple manifold, which we shall denote by $W(6)$, is obtained from the exterior $W$ of the Whitehead link by performing Dehn filling on its one boundary component with slope 6 under the standard meridian-longitude coordinates. In this paper, we shall show that $W(6)$ is the only simple manifold having two such Dehn fillings at distance 3 .

Theorem 1.2. Let $M$ be a simple manifold. If $M\left(r_{1}\right)$ is reducible and $M\left(r_{2}\right)$ contains a Klein bottle with $\Delta\left(r_{1}, r_{2}\right)=3$, then $M$ is homeomorphic to $W(6)$.

Corollary 1.3. Let $M$ be a simple manifold. If $M\left(r_{1}\right)$ is reducible and $M\left(r_{2}\right)$ is a Seifert fibered manifold over the 2-sphere with three exceptional fibers of orders $2,2, n$, then $\Delta\left(r_{1}, r_{2}\right) \leq 2$.

It is still unknown whether or not the upper bound 2 is the best possible.

\section{The intersection graphs}

From now on we assume that $M$ is a simple 3 -manifold with a torus boundary component $\partial_{0} M$ and that $r_{1}$ and $r_{2}$ are slopes on $\partial_{0} M$ of distance 3 apart such that $M\left(r_{1}\right)$ is reducible and $M\left(r_{2}\right)$ contains an essential torus or a Klein bottle.

Over all reducing spheres in $M\left(r_{1}\right)$ which intersect the attached solid torus $J_{1}$ in a family of meridian disks, we choose a 2-sphere $\widehat{F}_{1}$ so that $F_{1}=\widehat{F}_{1} \cap M$ has the minimal number, say $n_{1}$, of boundary components. Similarly let $\widehat{F}_{2}$ be either an essential torus or a Klein bottle in $M\left(r_{2}\right)$ which intersects the attached solid torus $J_{2}$ in a family of meridian disks, the number of which, say $n_{2}$, is minimal over all such surfaces and let $F_{2}=\widehat{F}_{2} \cap M$. Let $u_{1}, u_{2}, \ldots, u_{n_{1}}$ be the disks of $\widehat{F}_{1} \cap J_{1}$, labelled as they appear along $J_{1}$. Similarly let $v_{1}, v_{2}, \ldots, v_{n_{2}}$ be the disks of $\widehat{F}_{2} \cap J_{2}$. Then $F_{1}$ is an essential planar surface, and $F_{2}$ is an essential punctured torus or a punctured Klein bottle in $M$. We may assume that $F_{1}$ and $F_{2}$ intersect transversely and the number of components in $F_{1} \cap F_{2}$ is minimal over all such surfaces. Then no circle component of $F_{1} \cap F_{2}$ bounds a disk in either $F_{1}$ or $F_{2}$ and no arc component is boundary-parallel in either $F_{1}$ or $F_{2}$. The components of $\partial F_{i}$ are numbered $1,2, \ldots, n_{i}$ according to the labels

of the corresponding disks of $\widehat{F}_{i} \cap J_{i}$. We obtain a graph $G_{i}$ in $\widehat{F}_{i}$ by taking as the (fat) vertices of $G_{i}$ the disks in $\widehat{F}_{i} \cap J_{i}$ and as the edges of $G_{i}$ the arc components of $F_{1} \cap F_{2}$ in $F_{i}$. Each endpoint of an edge of $G_{i}$ has a label, that is, the number of the corresponding component of $\partial F_{j}, i \neq j$. Since each component of $\partial F_{i}$ intersects each component of $\partial F_{j}$ in $\Delta\left(=\Delta\left(r_{1}, r_{2}\right)=3\right)$ points, the labels $1,2, \ldots, n_{j}$ appear in order around each vertex of $G_{i}$ repeatedly $\Delta$ times. 
For a graph $G$, the reduced graph $\bar{G}$ of $G$ is defined to be the graph obtained from $G$ by amalgamating each family of parallel edges into a single edge. For an edge $\alpha$ of $\bar{G}$, the weight of $\alpha$, denoted by $w(\alpha)$, is the number of edges of $G$ represented by $\alpha$.

Although $F_{2}$ may be non-orientable, we can establish a parity rule. In fact, this is a natural generalization of the usual one. First, orient all components of $\partial F_{i}$ so that they are mutually homologous on $\partial_{0} M, i=1,2$. Let $e$ be an edge in $G_{i}$. Since $e$ is a properly embedded arc in $F_{i}$, it has a disk neighborhood $D$ in $F_{i}$ with $\partial D=a \cup b \cup c \cup d$, where $a$ and $c$ are arcs in $\partial F_{i}$ with induced orientation from $\partial F_{i}$. On $D$, if $a$ and $c$ have opposite directions, then $e$ is called positive, otherwise negative. See Figure 1. Then we have the following.

Parity rule. An edge is positive on one graph if and only if it is negative on the other graph.

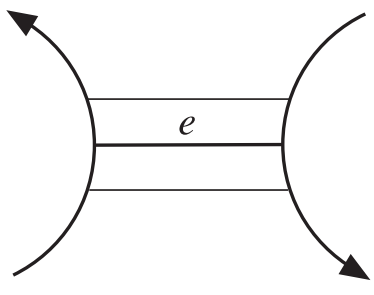

positive

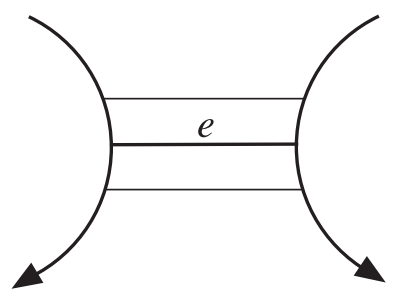

negative

\section{FiguRE 1}

Orient the core of $J_{i}$. If $\widehat{F}_{i}$ is orientable, we can give a sign to each vertex of $G_{i}$ according to the sign of its intersection with the core of $J_{i}$. Two vertices (possibly equal) of $G_{i}$ are called parallel if they have the same sign, otherwise antiparallel. A positive edge connects parallel vertices, while a negative one connects antiparallel vertices. Let $G_{i}^{+}$denote the subgraph of $G_{i}$ consisting of all the vertices and all the positive edges of $G_{i}$.

Let $G$ be $G_{1}$ or $G_{2}$ and let $x$ be a label of $G$. An $x$-edge is an edge of $G$ with label $x$ at one endpoint. An $x$-cycle is a cycle of positive $x$-edges which can be oriented so that the tail of each edge has label $x$. A cycle in $G$ is a Scharlemann cycle if it bounds a disk face, and the edges in the cycle are all positive and have the same label pair. If the label pair is $\{x, y\}$, then we refer to such a Scharlemann cycle as an $(x, y)$-Scharlemann cycle. In particular, a Scharlemann cycle of length 2 is called an $S$-cycle. An edge in $G$ is called level if its endpoints have the same label. A set of four parallel edges $\left\{e_{1}, e_{2}, e_{3}, e_{4}\right\}$ of $G_{2}$ is called an extended $S$-cycle if $\left\{e_{2}, e_{3}\right\}$ is an $S$-cycle and $e_{k}$ is adjacent to $e_{k+1}(k=1,2,3)$.

Lemma 2.1. (1) $G_{2}$ has no positive level edge.

(2) $G_{2}$ has no extended $S$-cycle.

(3) Suppose $\widehat{F}_{j}$ is not a Klein bottle. If $G_{i}$ has a Scharlemann cycle, $\widehat{F}_{j}$ is separating, $i \neq j$. 
(4) Any two Scharlemann cycles of $G_{2}$ have the same label set.

(5) Any positive edge $\alpha$ of $\bar{G}_{2}$ has $w(\alpha) \leq n_{1} / 2+1$.

(6) Any edge $\alpha$ of $\bar{G}_{2}$ has $w(\alpha)<n_{1}$.

(7) Let $\left\{e_{1}, \ldots, e_{k}\right\}$ be a set of parallel positive edges of $G_{2}$ with $e_{l}$ adjacent to $e_{l+1}(l=1, \ldots, k-1)$. If the sets of labels at two ends of $\left\{e_{1}, \ldots, e_{k}\right\}$ have a label in common, then either $\left\{e_{1}, e_{2}\right\}$ or $\left\{e_{k-1}, e_{k}\right\}$ forms an $S$ cycle. Moreover, the common label belongs to the label set of the S-cycle.

Proof. (1) By the parity rule a positive level edge in $G_{2}$ is a negative loop in $G_{1}$, which has a Möbius band neighborhood in $\widehat{F}_{1}$, contradicting that $\widehat{F}_{1}$ is a sphere. (2)-(4) follow from [23, Lemma 1.2], (5) and (6) follow from [23, Lemma 1.5], and (7) follows from (2),(4) and [4, Lemma 2.6.6].

Lemma 2.2. $n_{2}=2$ when $\widehat{F}_{2}$ is a torus, and $n_{2}=1$ when $\widehat{F}_{2}$ is a Klein bottle.

Proof. This is a part of the main result in [17].

\section{Klein bottle}

Throughout this section we assume that $\widehat{F}_{2}$ is a Klein bottle. Then $G_{2}$ has a single vertex $v$ by Lemma 2.2. The reduced graph $\bar{G}_{2}$ is a subgraph of the graphs shown in Figure 2. Whether $\bar{G}_{2}$ is a subgraph of the graph in Figure 2(a) or (b), there are three edge classes, $\alpha, \beta$ and $\gamma$. An edge in $G_{1}$ or $G_{2}$ is called an $\alpha$-edge, $\beta$-edge or $\gamma$-edge according as, being regarded as an edge in $G_{2}$, it lies in class $\alpha, \beta$ or $\gamma$. In $G_{2}$, all $\gamma$-edges are positive, while the others are negative.

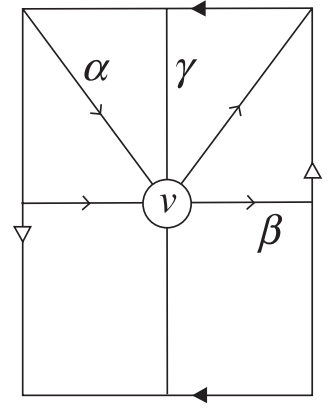

(a)

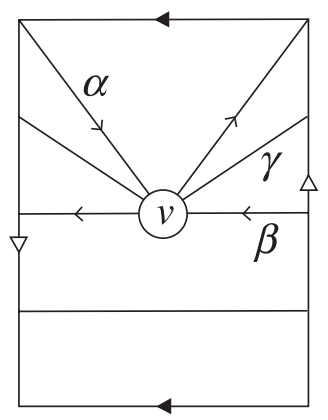

(b)

Figure 2

Lemma 3.1. The weights of the reduced edges $\alpha$ and $\beta$ in $\bar{G}_{2}$ are positive.

Proof. Assume $w(\alpha)=0$. By Lemma 2.1(5) and (6), $w(\beta)=n_{1}-1$ and $w(\gamma)=$ $n_{1} / 2+1$. If $\bar{G}_{2}$ is a subgraph of the graph in Figure $2(\mathrm{a})$, then $G_{2}$ has two positive level edges, contradicting Lemma 2.1(1). If $\bar{G}_{2}$ is a subgraph of the graph in Figure 2(b), then $G_{2}$ contains an $S$-cycle, so $\widehat{F}_{1}$ is separating and $n_{1}$ is even. However, for any $\beta$-edge in $G_{2}$, which is negative, the two labels at 
its endpoints have distinct parities. This contradicts the parity rule. Similarly, $w(\beta)>0$.

Thus the edges in $\bar{G}_{2}$ cut $F_{2}$ into one or two disks, so there is no circle component of $F_{1} \cap F_{2}$.

Orienting the negative edges in $G_{2}$ as shown in Figure 2, we can think of $G_{1}^{+}$ as a directed graph. If a disk face of $G_{1}^{+}$is bounded by a circuit of consistently oriented edges, we call it a cycle face. Throughout this section, let $I_{1}(\alpha)$ (resp. $I_{2}(\alpha)$ ) denote the shortest interval on $\partial v$ containing $\alpha$-edge endpoints at the head of $\alpha$ (resp. at the tail of $\alpha$ ). And similarly for $I_{1}(\beta)$ and $I_{2}(\beta)$.

Lemma 3.2. $G_{1}^{+}$has a cycle face.

Proof. First, assume that $\bar{G}_{2}$ is a subgraph of the graph in Figure 2(a). Then $w(\alpha)+w(\beta) \geq n_{1}$. Otherwise, $w(\alpha)+w(\beta)=n_{1}-1$ or $\leq n_{1}-2$. In the first case, the outermost edges in the family of $\gamma$-edges would be positive level edges, and in the latter case $w(\gamma) \geq n_{1} / 2+2$. Both are absurd by Lemma 2.1(1) and (5). Hence each label $x$ appears at least once on each of $I_{1}(\alpha) \cup I_{1}(\beta)$ and $I_{2}(\alpha) \cup I_{2}(\beta)$. This means that in $G_{1}^{+}$, each vertex $u_{x}$ of $G_{1}^{+}$has an edge pointing in and an edge pointing out. Starting at any vertex of $G_{1}^{+}$, one can construct a path through the oriented edges always consistent with orientations. Ultimately the path hits the same vertex to create a cycle. Among such cycles, an innermost one bounds a disk face of $G_{1}^{+}$and we are done.

Next, assume that $\bar{G}_{2}$ is a subgraph of the graph in Figure 2(b).

Claim. If $G_{1}^{+}$has a sink or source at a vertex $u_{x}$, then $x$ is a label of an $S$-cycle of $G_{2}$.

Proof. Suppose for example that $G_{1}^{+}$has a sink at $u_{x}$. We first show that $u_{x}$ is univalent in $G_{1}^{+}$. If $u_{x}$ were trivalent in $G_{1}^{+}$, then $u_{x}$ would have two (say) $\alpha$-edges pointing in. This means that label $x$ would appear twice on $I_{1}(\alpha)$, so $w(\alpha)>n_{1}$, contradicting Lemma 2.1(6). Thus $u_{x}$ has valency at most 2 in $G_{1}^{+}$. Suppose $u_{x}$ is bivalent in $G_{1}^{+}$. Then an $\alpha$-edge and a $\beta$-edge are incident to $u_{x}$ (otherwise, two (say) $\alpha$-edges would be incident, contradicting Lemma 2.1(6) as above). Since $u_{x}$ is trivalent in $G_{1}$, a $\gamma$-edge is incident to $u_{x}$ in $G_{1}$. Orient $\gamma$ so that its head lies between the tail of $\alpha$ and the head of $\beta$. Then in $G_{2}$, label $x$ appears at the heads of $\alpha, \beta$ and either at the head of $\gamma$ or at the tail of $\gamma$, say, at the head. Then $x$ appears twice at the heads of $\beta$ and $\gamma$, implying $w(\beta)+w(\gamma)>n_{1}$, and $x$ does not appear at the tails of $\beta$ and $\gamma$, implying $w(\beta)+w(\gamma)<n_{1}$. Two inequalities give a contradiction. Thus $u_{x}$ is univalent in $G_{1}^{+}$.

In $G_{1}$, two $\gamma$-edges are incident to $u_{x}$. In $G_{2}$, by Lemma 2.1(6), label $x$ appears at both ends of $\gamma$. By Lemma 2.1(7) the $\gamma$-edge family contains an $S$-cycle and $x$ is a label of this $S$-cycle.

Suppose $G_{1}^{+}$has no cycle face. Then $G_{1}^{+}$has a sink or source by [10, Lemma 2.3.1]. The above claim and [19, Lemma 2.3(1)] imply that there are exactly 
two sinks and sources in total. Let $u_{x}$ and $u_{y}$ be vertices of $G_{1}$ at which these sinks and sources occur (then labels $x, y$ form a label pair of an $S$-cycle in $G_{2}$ ). Then two $\gamma$-edges running from $u_{x}$ to $u_{y}$ divide $\widehat{F}_{1}$ into two disks and each of them contains neither sink nor source in its interior. The two disks have the same number of vertices of $G_{1}$ in their interiors by [21, Lemma 2.1]. One can choose a disk whose interior contains no positive edge incident to a sink. Then there would be a cycle face in the disk.

Orient all components of $\partial F_{1}=\left\{\partial u_{1}, \ldots, \partial u_{n_{1}}\right\}$ homologously on $\partial_{0} M$ and orient $\partial F_{2}=\partial v$. Let $u \in\left\{u_{1}, \ldots, u_{n_{1}}\right\}$. If $P$ and $Q$ are two points in $\partial u \cap \partial v$, denote by $\mu_{1}(P, Q)$ (resp. $\left.\mu_{2}(P, Q)\right)$ the arc in $\partial u$ (resp. $\left.\partial v\right)$ going from $P$ to $Q$ with respect to the chosen orientation. As in $\left[6\right.$, p.1720] we define $\tau_{i}(P, Q)=$ $\left|\mu_{i}(P, Q) \cap \partial F_{j}\right|-1(\{i, j\}=\{1,2\})$.

Lemma 3.3. Let $u, u^{\prime} \in\left\{u_{1}, \ldots, u_{n_{1}}\right\}$. Suppose $P, Q \in \partial u \cap \partial v$ and $R, S \in$ $\partial u^{\prime} \cap \partial v$. If $\tau_{1}(P, Q)=\tau_{1}(R, S)$, then $\tau_{2}(P, Q)=\tau_{2}(R, S)$.

Proof. This follows from [6, Lemma 2.4].

Lemma 3.4. Let $f$ be a cycle face with vertices $u_{x_{1}}, \ldots, u_{x_{n}}$ and with corners $\lambda_{i}$ at $u_{x_{i}}$, i.e. the intervals $f \cap u_{x_{i}}$ on $\partial u_{x_{i}}$. Let $\partial^{1} \lambda_{i}$ be one endpoint of $\lambda_{i}$ at the head of an oriented edge of $f$ and $\partial^{2} \lambda_{i}$ the other endpoint (automatically at the tail of another edge of $f)$. Then we have $\tau_{2}\left(\partial^{1} \lambda_{1}, \partial^{2} \lambda_{1}\right)=\cdots=\tau_{2}\left(\partial^{1} \lambda_{n}, \partial^{2} \lambda_{n}\right)$.

Proof. Since $u_{x_{i}}$ 's are all parallel, an orientation of $F_{1}$ induces orientations of $\partial u_{x_{i}}$ 's which are mutually homologous on $\partial_{0} M$, so $\tau_{1}\left(\partial^{1} \lambda_{1}, \partial^{2} \lambda_{1}\right)=\cdots=$ $\tau_{1}\left(\partial^{1} \lambda_{n}, \partial^{2} \lambda_{n}\right)$. By Lemma 3.3 we have $\tau_{2}\left(\partial^{1} \lambda_{1}, \partial^{2} \lambda_{1}\right)=\cdots=\tau_{2}\left(\partial^{1} \lambda_{n}, \partial^{2} \lambda_{n}\right)$.

Proposition 3.5. $\bar{G}_{2}$ is a subgraph of the graph in Figure $2(b)$.

Proof. Assume for contradiction that $\bar{G}_{2}$ is a subgraph of the graph in Figure $2(\mathrm{a})$. Let $f$ be a cycle face of $G_{1}^{+}$guaranteed by Lemma 3.2 and $u_{x_{1}}, \ldots, u_{x_{n}}$ the vertices of $f$. Let $\lambda_{i}$ be the corner of $f$ at $u_{x_{i}}$ with one endpoint, $\partial^{1} \lambda_{i}$, at the head of an oriented edge of $f$ and the other, $\partial^{2} \lambda_{i}$, at the tail of another edge of $f$. On $\partial v$, choose the shortest interval $I_{j}$ such that $\left\{\partial^{j} \lambda_{1}, \ldots, \partial^{j} \lambda_{n}\right\} \subset I_{j}$ for $j=1,2$. Since $\left\{\partial^{j} \lambda_{1}, \ldots, \partial^{j} \lambda_{n}\right\} \subset I_{j}(\alpha) \cup I_{j}(\beta), I_{1} \cap I_{2}=\emptyset$. Label $x_{1}, \ldots, x_{n}$ so that $\partial I_{1}=\left\{\partial^{1} \lambda_{1}, \partial^{1} \lambda_{n}\right\}$. Using Lemma 3.4, one can verify that $\partial I_{2}=\left\{\partial^{2} \lambda_{1}, \partial^{2} \lambda_{n}\right\}$. Hence $I_{1} \cup I_{2} \cup \lambda_{1} \cup \lambda_{n}$ bounds a disk $D$ on $\partial_{0} M$. As below the proof of [11, Claim 7.5], one can use $D$ and $f$ to construct a new Klein bottle in $M\left(r_{2}\right)$, on which the core of $J_{2}$ can be isotoped to lie. This implies that $M$ contains a properly embedded Möbius band and hence fails to be simple.

By Lemma 3.2, $G_{1}^{+}$has a disk face $f$ bounded by a cycle of consistently oriented edges $e_{1}, \ldots, e_{n}$, labelled so that the head of $e_{i}$ is adjacent to the tail of $e_{i+1}$ modulo $n$. The edges $e_{1}, \ldots, e_{n}$ do not totally belong to one edge class, $\alpha$ or $\beta$, since otherwise, the argument in $[9$, Section 5$]$ would show that $M$ contains a cable space. 
Lemma 3.6. $n$ is even and $\left\{e_{1}, \ldots, e_{n}\right\}$ is an alternating sequence of $\alpha$-edges and $\beta$-edges.

Proof. If $n=2$, it is obvious, so we assume $n>2$. Assume for contradiction that $e_{1}, e_{2}$ are $\alpha$-edges and $e_{3}$ is a $\beta$-edge. Let $u_{x_{1}}$ be the vertex to which $e_{1}$ and $e_{2}$ are incident and let $u_{x_{2}}$ be the vertex to which $e_{2}$ and $e_{3}$ are incident. Let $\lambda_{i}$ be the corner of $f$ at $u_{x_{i}}$ with endpoints $\partial^{j} \lambda_{i}=e_{i+j-1} \cap u_{x_{i}}(i, j=1,2)$. Then in $G_{2}$, the points $\partial^{1} \lambda_{1}, \partial^{2} \lambda_{1}, \partial^{1} \lambda_{2}, \partial^{2} \lambda_{2}$ are on $I_{1}(\alpha), I_{2}(\alpha), I_{1}(\alpha), I_{2}(\beta)$, respectively.

Orient $\partial v$ clockwise. By Lemma $3.4 \tau_{2}\left(\partial^{1} \lambda_{1}, \partial^{2} \lambda_{1}\right)=\tau_{2}\left(\partial^{1} \lambda_{2}, \partial^{2} \lambda_{2}\right)$. Recall that $\bar{G}_{2}$ is a subgraph of the graph in Figure 2(b). From the fact that the points $\partial^{1} \lambda_{1}, \partial^{2} \lambda_{1}, \partial^{1} \lambda_{2}, \partial^{2} \lambda_{2}$ are respectively on $I_{1}(\alpha), I_{2}(\alpha), I_{1}(\alpha), I_{2}(\beta)$, one can obtain two inequalities $\tau_{2}\left(\partial^{1} \lambda_{1}, \partial^{2} \lambda_{1}\right) \leq 2 w(\alpha)<2 n_{1}$ and $\tau_{2}\left(\partial^{1} \lambda_{2}, \partial^{2} \lambda_{2}\right)>$ $w(\alpha)+w(\beta)+w(\gamma)=3 n_{1} / 2$. Since $\partial^{1} \lambda_{i}, \partial^{2} \lambda_{i}$ are labelled $x_{i}, \tau_{2}\left(\partial^{1} \lambda_{1}, \partial^{2} \lambda_{1}\right)=$ $n_{1}$ and $\tau_{2}\left(\partial^{1} \lambda_{2}, \partial^{2} \lambda_{2}\right)=2 n_{1}$, giving a contradiction.

Lemma 3.7. $f$ is a bigon.

Proof. Assume that $n=2 m>2$. Label the vertices of $f u_{x_{1}}, u_{y_{1}}, \ldots, u_{x_{m}}, u_{y_{m}}$ along $\partial f$ so that an $\alpha$-edge (resp. $\beta$-edge) is incident to $u_{x_{i}}$ (resp. $u_{y_{i}}$ ) at its head. See Figure 3. Then each label $x_{i}$ (resp. $y_{i}$ ) appears once on each of $I_{1}(\alpha)$ and $I_{2}(\beta)$ (resp. $I_{1}(\beta)$ and $I_{2}(\alpha)$ ). Since $w(\alpha), w(\beta)<n_{1}$, any label cannot occur twice on each interval.

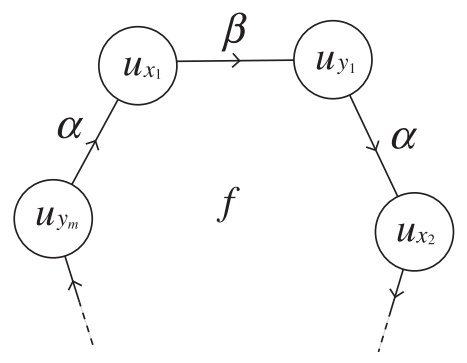

FiguRE 3

Orient $\partial v$ clockwise. Relabelling $x_{i}$ 's (and correspondingly $y_{i}$ 's), if necessary, we may assume that among $x_{i}$ 's, $x_{1}$ appears first on $I_{1}(\alpha)$ with respect to the orientation of $\partial v$. Then $x_{1}$ precedes other $x_{i}$ 's on $I_{2}(\beta)$ by Lemma 3.4. The vertices $u_{x_{i}}$ and $u_{y_{i}}$ are connected by a $\beta$-edge for each $i$. Since $\beta$-edges are negative in $G_{2}, y_{1}$ also precedes other $y_{i}$ 's on $I_{1}(\beta)$. Again by Lemma 3.4, $y_{1}$ precedes other $y_{i}$ 's on $I_{2}(\alpha)$. In particular, $y_{m}$ follows $y_{1}$ on $I_{2}(\alpha)$. Since $u_{y_{m}}, u_{x_{1}}$ and $u_{y_{1}}, u_{x_{2}}$ are connected by $\alpha$-edges, respectively and since $\alpha$-edges are negative in $G_{2}, x_{1}$ follows $x_{2}$ on $I_{1}(\alpha)$. This contradicts the choice of $x_{1}$.

Without loss of generality we may assume that the labels around $v$ are ordered in the clockwise direction. Then one can see that if an $\alpha$-edge (resp. a $\beta$-edge) in $G_{2}$ has label $x$ at its head, then its tail is labelled $x+w(\alpha)($ resp. $x+w(\beta))$ modulo $n_{1}$. 
By Lemmas 3.6 and 3.7, $f$ has exactly two vertices $u_{x}, u_{y}$ along with two edges $e_{\alpha}, e_{\beta}\left(e_{i}\right.$ is an $i$-edge, $\left.i=\alpha, \beta\right)$ such that $e_{\alpha}$ and $e_{\beta}$ are incident to $u_{x}$ and $u_{y}$ at their heads, respectively. Then $y \equiv x+w(\alpha), x \equiv y+w(\beta)\left(\bmod n_{1}\right)$, so $w(\alpha)+w(\beta) \equiv 0$. Since $0<w(\alpha)+w(\beta)<2 n_{1}$, we get $w(\alpha)+w(\beta)=n_{1}$. Thus $w(\gamma)=n_{1} / 2$. By Lemma 2.1(7), either the $\gamma$-edge family in $G_{2}$ contains an $S$-cycle or the sets of labels at its two ends are disjoint.

Suppose that the $\gamma$-edge family contains an $S$-cycle $\sigma$ with label pair $\{1,2\}$, say. Then $\sigma$ consists of either the first two edges or the last two edges of the family. By examining the labels around the vertex $v$, one can see that either $w(\alpha)=n_{1} / 2-1$ or $w(\beta)=n_{1} / 2-1$ holds. We conclude that the three numbers $w(\alpha), w(\beta), w(\gamma)$ cannot be all even.

We shall rechoose $\widehat{F}_{1}$ to rule out this case. Let $f$ be the disk face bounded by $\sigma$. The edges of $\sigma$ cut $\widehat{F}_{1}-\operatorname{Int}\left(u_{1} \cup u_{2}\right)$ into two disks, $D^{\prime}$ and $D^{\prime \prime}$, say. Put $D=D^{\prime} \cup u_{1}$. Then $D$ contains $n_{1} / 2$ fat vertices by [21, Lemma 2.1], and $f \cup D$ is a Möbius band whose boundary bounds a disk $B$ on $\partial H$, where $H$ is the part of $J_{1}$ between the consecutive vertices $u_{1}$ and $u_{2}$. After being slightly pushed off $H, \widehat{P}=f \cup D \cup B$ becomes a projective plane which intersects $J_{1}$ in $n_{1} / 2$ meridian disks. For a thin $I$-bundle neighborhood $N$ of $\widehat{P}$ in $M\left(r_{1}\right)$, its boundary is a reducing sphere intersecting $J_{1}$ in $n_{1}$ meridian disks. Using $\partial N$ instead of $\widehat{F}_{1}$, we obtain a new graph pair $G_{1}, G_{2}$, where each edge of $\bar{G}_{2}$ has an even weight by the $I$-bundle structure of $N$. In particular, the above observation shows that $G_{2}$ cannot have an $S$-cycle.

Therefore we may assume that the label sets at two ends of the $\gamma$-edge family are disjoint and hence $w(\alpha)=w(\beta)=w(\gamma)=n_{1} / 2$.

Lemma 3.8. $n_{1}=4$ and $\widehat{F}_{1}$ is separating.

Proof. Since $\Delta n_{1}=3 n_{1}=2(w(\alpha)+w(\beta)+w(\gamma)), n_{1}$ is even. Let $n_{1}=2 m$. We may assume that the labels around $v$ are as shown in Figure 4. Notice that $n_{1} / 2=m$ is also even, for otherwise the central edge in the $\gamma$-edge family would have the same label pair as the central edge in the $\alpha$-edge family and they would form an orientation-reversing loop in $\widehat{F}_{1}$.

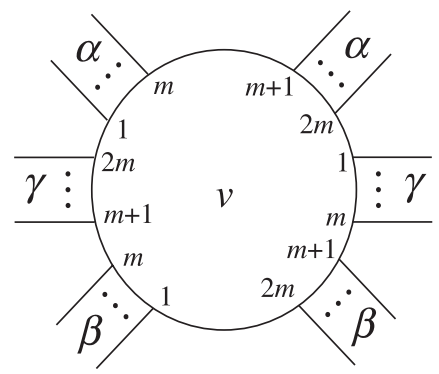

FiguRE 4

Let $e_{i}$ (resp. $e_{i}^{\prime}$ ) be a $\gamma$-edge (resp. an $\alpha$-edge) in $G_{2}$ with label $i$ at one endpoint, $i=1, \ldots, m$. Let $D_{i}$ be the disk in $F_{2}$, realizing the parallelism of $e_{i}$ 
and $e_{m-i+1}$ with $\partial D_{i}=e_{i} \cup a_{i} \cup e_{m-i+1} \cup b_{i}$, where $a_{i}$ is an arc on $\partial v$ from label $i$ to $m-i+1$. And similarly for $D_{i}^{\prime}, a_{i}^{\prime}, b_{i}^{\prime}$. The four edges $e_{i}, e_{m-i+1}, e_{i}^{\prime}, e_{m-i+1}^{\prime}$ together with vertices $u_{i}, u_{m-i+1}, u_{m+i}, u_{2 m-i+1}$ form a loop $\sigma_{i}$ on $\widehat{F}_{1}$. These four vertices divide $J_{1}$ into four parts. Let $U_{i}$ (resp. $V_{i}$ ) be the part of $J_{1}$ between $u_{i}$ and $u_{m-i+1}$ (resp. $u_{m+i}$ and $\left.u_{2 m-i+1}\right)$. A regular neighborhood of $\widehat{F}_{1} \cup U_{m / 2} \cup V_{m / 2} \cup D_{m / 2} \cup D_{m / 2}^{\prime}$ in $M\left(r_{1}\right)$ is a punctured projective space with two boundary spheres one of which is parallel to $\widehat{F}_{1}$ and the other intersects $J_{1}$ in fewer components than $\widehat{F}_{1}$, so bounds a 3 -ball. Hence $\widehat{F}_{1}$ is separating.

Now assume that $n_{1}>4$. Among the loops $\sigma_{i}$ 's, choose an innermost one, say, $\sigma_{k}$. Then $a_{k}$ and $a_{k}^{\prime}$ are properly imbedded essential arcs in the annulus $\partial U_{k}-\operatorname{Int}\left(u_{k} \cup u_{m-k+1}\right)$, and $I_{2}(\beta)$ intersects the annulus in a spanning arc. The arcs $a_{k}$ and $a_{k}^{\prime}$ cut the annulus into two disks and one can choose a component $B$ disjoint from $I_{2}(\beta)$. Similarly after cutting the annulus $\partial V_{k}-\operatorname{Int}\left(u_{m+k} \cup\right.$ $\left.u_{2 m-k+1}\right)$ along the arcs $b_{k}$ and $b_{k}^{\prime}$, one can choose a component $B^{\prime}$ disjoint from $I_{1}(\beta)$. Then $D_{k} \cup D_{k}^{\prime} \cup B \cup B^{\prime}$ is a Möbius band whose boundary bounds a disk in $\widehat{F}_{1}$ containing exactly two vertices, either $\left\{u_{k}, u_{m+k}\right\}$ or $\left\{u_{m-k+1}, u_{2 m-k+1}\right\}$. Hence we can find a projective plane in $M\left(r_{1}\right)$ intersecting the core of $J_{1}$ in two points. The boundary of a thin regular neighborhood of this projective plane is a reducing sphere of $M\left(r_{1}\right)$, intersecting $J_{1}$ in fewer components than $\widehat{F}_{1}$, which contradicts our choice of $\widehat{F}_{1}$ at the beginning of Section 2 .

Proof of Theorem 1.2. By Lemma 3.8 and the argument just above it, $G_{2}$ is uniquely determined as illustrated in Figure $5(\mathrm{a})$. Let $A, B, C, D, E, F$ be the edges of $G_{2}$ as shown in Figure 5(a). The correspondence between the edges of $G_{1}$ and $G_{2}$ uniquely determines $G_{1}$ up to a homeomorphism of the underlying sphere $\widehat{F}_{1}$, as shown in Figure $5(\mathrm{~b})$.

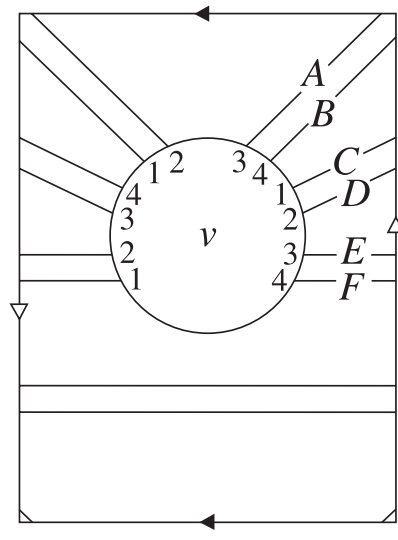

(a)

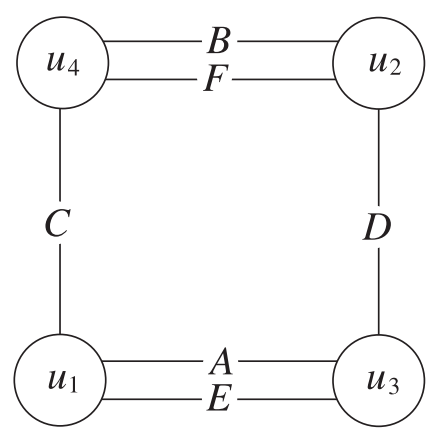

(b)

FIGURE 5

The graph $G_{2}$ has two trigons $f_{1}, f_{2}$ bounded by $A, B, C$ and $D, E, F$, respectively. Let $g_{1}, g_{2}$ be bigons of $G_{2}$ bounded by $A, B$ and $C, D$, respectively. 
These trigons and bigons lie on the opposite sides of $\widehat{F}_{1}$. Let $X$ be a regular neighborhood of $\partial_{0} M \cup F_{1} \cup f_{1} \cup f_{2} \cup g_{1} \cup g_{2}$ in $M$. Then one can verify that $\partial X$ consists of two spheres. Capping off these spheres with 3-balls gives $M$. This shows that $M$ is uniquely determined by the pair of the graphs in Figure 5. Hence $M=W(6)$.

Proof of Corollary 1.3. Since $M\left(r_{2}\right)$ contains a Klein bottle, $\Delta\left(r_{1}, r_{2}\right) \leq 3$ by [19, Theorem 1.1]. Assume for contradiction that $\Delta\left(r_{1}, r_{2}\right)=3$. Then $M=$ $W(6)$ by Theorem 1.2.

Note that $M(\infty)=L(6,1)$ and $M(2)$ is a small Seifert fibered space with a finite fundamental group. See [3, Example 7.8]. We have $\Delta\left(r_{1}, \infty\right) \leq 1$ and $\Delta\left(r_{1}, 1\right) \leq 1$ by $\left[3\right.$, Theorem 1.2(1)] and [12, Theorem 1.2]. Hence $r_{1}=0,1$ or 2. However, $M(2)$ is irreducible. The slope 0 is a boundary slope of an essential once-punctured torus in $M$, which extends to an essential torus in $M(0)$, and $\operatorname{dim} H_{1}(M ; \mathbb{Q})=1$. The conclusions (ii), (iii) and (iv) in [4, Theorem 2.0.3] do not hold for $M$ and the slope 0 , so $M(0)$ is irreducible. Therefore $r_{1}=1$. By $\left[2\right.$, Theorem 1.5(1)] we have $\Delta\left(r_{2}, \infty\right)=1$ and hence the assumption $\Delta\left(r_{1}, r_{2}\right)=3$ yields $r_{2}=-2$ or 4 . However, $M(-2)$ is hyperbolic by [7, Example (5)]. Therefore $r_{2}=4$.

\section{Torus}

Throughout this section we assume that $\widehat{F}_{2}$ is a torus. Then $G_{2}$ has exactly two vertices, $v_{1}$ and $v_{2}$, by Lemma 2.2. We may assume that $M\left(r_{2}\right)$ is irreducible and boundary-irreducible by $[12,20]$. By Lemma 2.1(4), we also assume that $G_{2}$ has only $(1,2)$-Scharlemann cycles if it has Scharlemann cycles.

Lemma 4.1. The vertices of $G_{2}$ are antiparallel.

Proof. Assume that $v_{1}$ and $v_{2}$ are parallel. Then all the edges of $G_{2}$ are positive. For a label $x \neq 1,2$, consider the subgraph $\Gamma$ of $G_{2}$ consisting of all vertices and all $x$-edges of $G_{2}$. Let $V, E$ and $F$ be the number of vertices, edges and disk faces of $\Gamma$, respectively. Since $V<E$, we have $0=\chi\left(\widehat{F}_{2}\right) \leq V-E+F<F$, so $\Gamma$ contains a disk face, which is an $x$-face in $G_{2}$ in terms of [17]. This contradicts [17, Theorem 4.4].

The graph $G_{2}$ has at most 6 edge classes, which we call $\alpha, \beta, \gamma, \delta, \varepsilon, \varepsilon^{\prime}$ as illustrated in Figure 6. See [6, Lemma 5.2]. An edge in $G_{1}$ or $G_{2}$ is called an $\eta$-edge if, being regarded as an edge in $G_{2}$, it lies in class $\eta, \eta \in\left\{\alpha, \beta, \gamma, \delta, \varepsilon, \varepsilon^{\prime}\right\}$. An edge in $G_{1}$ or $G_{2}$ is called of type 1 if it is an $\alpha$-edge or a $\beta$-edge, and of type 2 if it is a $\gamma$-edge or a $\delta$-edge. The $\varepsilon$-edges and $\varepsilon^{\prime}$-edges are positive in $G_{2}$, while the others are negative by Lemma 4.1. Since $v_{1}$ and $v_{2}$ have the same valency, we have $w(\varepsilon)=w\left(\varepsilon^{\prime}\right)$. Without loss of generality we assume that the ordering of the labels around $v_{1}$ is anticlockwise, while the ordering around $v_{2}$ is clockwise.

Lemma 4.2. Let $x(\neq 1,2)$ be a label of $G_{2}$. Then there exist an edge of type 1 and an edge of type 2 incident to $v_{i}$ with label $x, i=1,2$. 


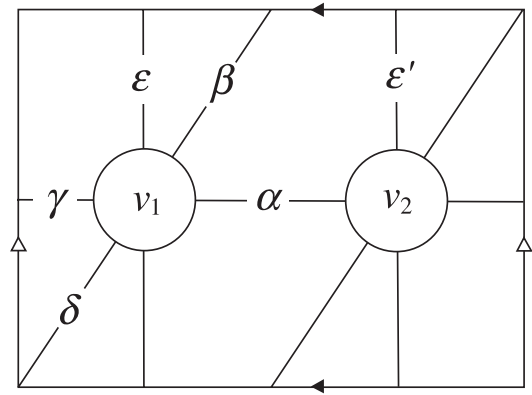

FiguRe 6

Proof. Let $\varepsilon=\left\{e_{1}, \ldots, e_{w(\varepsilon)}\right\}, \varepsilon^{\prime}=\left\{e_{1}^{\prime}, \ldots, e_{w\left(\varepsilon^{\prime}\right)}^{\prime}\right\}$ as shown in Figure 7 . There are two cases.

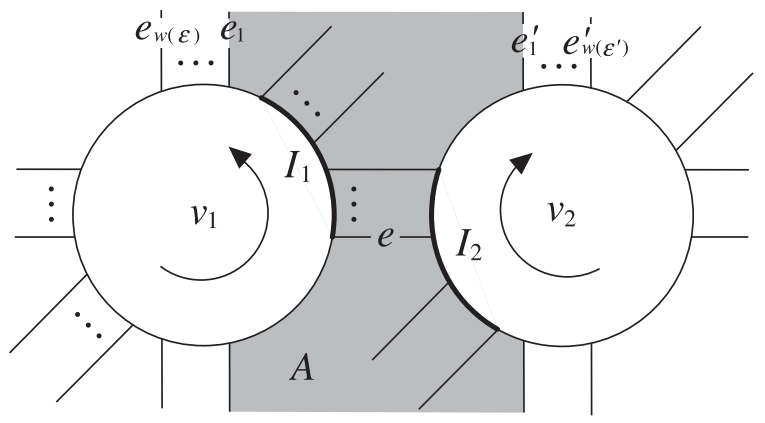

FiguRE 7

Case I. The sets of labels at two ends of $\varepsilon$ have a label in common. By Lemma 2.1(7) we may assume that $\left\{e_{1}, e_{2}\right\}$ is an $S$-cycle with label pair $\{1,2\}$. Let $I_{i}$ be the shortest interval on $\partial v_{i}$ containing the endpoints of the edges of type 1 at $v_{i}, i=1,2$. Since $I_{1}$ and $I_{2}$ have the same number of edge endpoints, $\left\{e_{1}^{\prime}, e_{2}^{\prime}\right\}$ is also an $S$-cycle with label pair $\{1,2\}$. Then $w(\alpha)+w(\beta)=n_{1}-2$ or $2 n_{1}-2$. In the first case for any label $x \neq 1,2$, exactly one edge of type 1 is incident to $v_{i}$ with label $x, i=1,2$. On the other hand, since $w(\varepsilon) \leq n_{1} / 2+1$ by Lemma $2.1(5), w(\gamma)+w(\delta)=3 n_{1}-w(\alpha)-w(\beta)-2 w(\varepsilon) \geq n_{1}$. This means that for any label $x$, an edge of type 2 is incident to $v_{i}$ with label $x, i=1,2$, so we have the desired result. In the latter case $w(\alpha)=w(\beta)=n_{1}-1$ by Lemma 2.1(6). Let $e$ be the lowest $\alpha$-edge as in Figure 7. Since $\left\{e_{1}, e_{2}\right\}$ and $\left\{e_{1}^{\prime}, e_{2}^{\prime}\right\}$ are $(1,2)$-S-cycles, the point $e \cap \partial v_{1}$ is labelled 3 , while the point $e \cap \partial v_{2}$ is labelled 2. Hence $e$ connects vertices $u_{2}$ and $u_{3}$ in $G_{1}$. Since $G_{2}$ contains $S$-cycles, $\widehat{F}_{1}$ is separating by Lemma $2.1(3)$, so $u_{2}$ and $u_{3}$ are antiparallel. Therefore the edge $e$ is negative in both graphs $G_{1}$ and $G_{2}$, which is impossible by the parity rule.

Case II. The sets of labels at two ends of $\varepsilon$ are disjoint. Suppose, for example, that no edge of type 1 is incident to $v_{1}$ with label $x \neq 1,2$, that is, the label $x$ does 
not appear on $I_{1}$. Since $w(\gamma), w(\delta)<n_{1}$ by Lemma 2.1(6), $w(\alpha)+w(\beta)+2 w(\varepsilon)=$ $3 n_{1}-w(\gamma)-w(\delta)>n_{1}$, so $x$ appears at one end of $\varepsilon$, say, at the bottom of $v_{1}$ (then $x$ does not appear at the top of $v_{1}$ ). Let $y$ be the label of the point in $\partial v_{1} \cap e_{w(\varepsilon)}$ at the bottom of $v_{1}$ ( $y$ is possibly equal to $x$ ). Then $y$ does not appear on $I_{1}$, otherwise $x$ must appear at the end of $\varepsilon$ at the top of $v_{1}$. Since $w(\alpha)+w(\beta)+2 w(\varepsilon)>n_{1}, y$ must appear at the end of $\varepsilon$ at the top of $v_{1}$. This contradicts our assumption.

In particular, we have $w(\alpha)+w(\beta)>0$ and $w(\gamma)+w(\delta)>0$. And if $w(\varepsilon)=$ $w\left(\varepsilon^{\prime}\right)=0$, then we have $w(\alpha), w(\beta), w(\gamma), w(\delta)>0$ by Lemma 2.1(6). Thus the reduced edges in $\bar{G}_{2}$ cut $F_{2}$ into disks, so $F_{1} \cap F_{2}$ has no circle component.

Proposition 4.3. $G_{1}^{+}$contains a connected subgraph $\Lambda$ satisfying that

(1) for all vertices $u_{x}$ of $\Lambda$ but at most one vertex, there are an edge of type 1 and an edge of type 2 in $\Lambda$ which are incident to $u_{x}$ with label $i$ for each $i=1,2$;

(2) for the other vertex $u_{x_{0}}$, if it exists, there are two edges in $\Lambda$ incident to $u_{x_{0}} ;$ and

(3) there is a disk $D_{\Lambda} \subset \widehat{F}_{1}$ such that $D_{\Lambda} \cap G_{1}^{+}=\Lambda$.

The vertex $u_{x_{0}}$ is called an exceptional vertex of $\Lambda$.

Proof. Let $\Gamma$ be an extremal component of $G_{1}^{+}$. That is, $\Gamma$ is a component of $G_{1}^{+}$ having a disk support $D$ such that $D \cap G_{1}^{+}=\Gamma$.

Recall that all the Scharlemann cycles in $G_{2}$ are $(1,2)$-Scharlemann cycles. If $G_{2}$ contains a Scharlemann cycle, then the vertices $u_{1}$ and $u_{2}$ are antiparallel and hence they belong to distinct components of $G_{1}^{+}$. So, we may assume that $\Gamma$ does not contain $u_{2}$ in this case.

A vertex of $\Gamma$ is a cut vertex if it splits $\Gamma$ into more components. If $\Gamma$ has no cut vertex, we take $\Gamma$ as $\Lambda$. Then all vertices of $\Lambda$, possibly except one (when $u_{1}$ is contained in $\Lambda$ ), satisfy the property (1) by Lemma 4.2 .

Suppose that $\Gamma$ has a cut vertex. If $u_{1}$ is a single cut vertex of $\Gamma$, cut $\Gamma$ off at $u_{1}$ and take any component as $\Lambda$, and then all vertices of $\Lambda$ but $u_{1}$ satisfy the property (1) again by Lemma 4.2. Otherwise, after cutting $\Gamma$ off at all cut vertices we can find a component, which does not contain $u_{1}$, with a single cut vertex. We take this component as $\Lambda$ and then the cut vertex may be the exceptional vertex of $\Lambda$, while the other vertices of $\Lambda$ satisfy the property (1) by Lemma 4.2 .

Let $D_{\Lambda}$ be a disk support of $\Lambda$ as given in Proposition 4.3. A vertex of $\Lambda$ is a boundary vertex if there is an arc connecting it to $\partial D_{\Lambda}$ whose interior is disjoint from $\Lambda$, and an interior vertex otherwise.

Each face of $\Lambda$ is a disk, for $\Lambda$ is connected. Since $G_{1}$ has only two labels, $\{1,2\}$, each edge of $\Lambda$ has label 1 at one endpoint and label 2 at the other. Hence every face of $\Lambda$ determines a Scharlemann cycle of $G_{1}$. By Lemma 2.1(3) 
$\widehat{F}_{2}$ divides $M\left(r_{2}\right)$ into a black side $X$ and a white side $Y$. A face of $G_{1}$ is black or white according as it lies in $X$ or $Y$.

From now on we assume that $\partial_{1} M=\partial M-\partial_{0} M$ is a torus and eventually we will get a contradiction. Assume that $Y$ contains $\partial_{1} M$. By Theorem 1.2 we may also assume that $M\left(r_{2}\right)$ contains no Klein bottle.

Lemma 4.4. The edges of a face in $G_{1}^{+}$cannot lie in a disk in $\widehat{F}_{2}$.

Proof. At the beginning of this section we assumed that $M\left(r_{2}\right)$ is irreducible. Hence the statement follows from the proof of [11, Lemma 3.1].

Let $H_{X}=J_{2} \cap X$ and $H_{Y}=J_{2} \cap Y$.

Lemma 4.5. For any white face of $G_{1}^{+}$, its edges cannot lie in an annulus in $\widehat{F}_{2}$.

Proof. Suppose to the contrary that $G_{1}^{+}$contains a white face $f$ whose edges lie in an annulus $A$ in $\widehat{F}_{2}$. By Lemma 4.4, $A$ is essential in $\widehat{F}_{2}$. Let $N$ be a regular neighborhood of $A \cup H_{Y} \cup f$ in $Y$. Then $\partial N$ is a torus, and $T=(\partial N-\operatorname{Int} A) \cup$ $\left(\widehat{F}_{2}-A\right)$ is a torus disjoint from $J_{2}$, so it must be inessential in $M\left(r_{2}\right)$. If $T$ were compressible, a compression would result in a sphere separating $\widehat{F}_{2}$ and $\partial_{1} M$, and hence a reducing sphere of $M\left(r_{2}\right)$. This contradicts our assumption that $M\left(r_{2}\right)$ is irreducible. Suppose $T$ is parallel to $\partial_{1} M$. Then there is an annulus $A^{\prime}$ in the region between the two tori $T$ and $\partial_{1} M$ such that one component of $\partial A^{\prime}$ lies in $\widehat{F}_{2}-A$ and the other component lies in $\partial_{1} M$. The circle $A^{\prime} \cap \partial_{1} M$ is an essential curve on $\partial_{1} M$, otherwise $\widehat{F}_{2}$ would be compressible. Surgering $\widehat{F}_{2}$ along $A^{\prime}$ gives a properly embedded annulus $A^{\prime \prime}$ in $M\left(r_{2}\right)$. Since $\widehat{F}_{2}$ is essential in $M\left(r_{2}\right)$, so is $A^{\prime \prime}$. Our assumption $\Delta\left(r_{1}, r_{2}\right)=3$ contradicts [22, Theorem 5.1].

Lemma 4.6. $\Lambda$ contains a face bounded only by edges of type 1 or only by edges of type 2 .

Proof. Let $u_{x}$ be the exceptional vertex of $\Lambda$, if it exists, and any vertex of $\Lambda$ otherwise. Without loss of generality we may assume that an edge $e$ of type 1 is incident to $u_{x}$ with label 1 . Orient every edge of type 1 from the endpoint with label 1 to the other. Then by Proposition 4.3 any non-exceptional vertex of $\Lambda$ has an edge pointing in and an edge pointing out. Starting with $e$, one can construct a path through the oriented edges of type 1 always consistent with orientations. Ultimately the path hits the same vertex to create a cycle. This shows that $\Lambda$ contains cycles of oriented edges of type 1 . Choose an innermost cycle $\sigma$. Then $\sigma$ bounds a disk $D$ in $\widehat{F}_{1}$ with no vertex in its interior. If $D$ has no edge of $\Lambda$ in its interior, then we are done. Otherwise, all the edge in $\operatorname{Int} D$ are of type 2. Since $\sigma$ is a 1-cycle, some of these edges bound a desired face of $\Lambda$.

Lemma 4.7. Let $f$ be an $n$-sided face of $G_{1}^{+}, n=2$ or 3 . Then the edges of $f$ lie in an essential annulus, $A$, in $\widehat{F}_{2}, f$ is black, and $X$ is a Seifert fibered space 
over the disk with two exceptional fibers one of which has order $n$. The core of $A$ is a Seifert fiber.

Proof. This follows from [11, Lemma 3.7 and Theorem 3.8] and Lemma 4.5.

Note that each of non-exceptional boundary vertices of $\Lambda$ has valency at least 4 in $\Lambda$ by the property (1) of Proposition 4.3 .

Lemma 4.8. $\Lambda$ contains a black bigon.

Proof. Let $V, E$ and $F$ be the number of vertices, edges and faces of $\Lambda$, respectively. Let $V_{b}, V_{i}$ and $V_{e}$ denote the number of boundary vertices, interior vertices and exceptional vertex of $\Lambda$, respectively. Then $V=V_{b}+V_{i}$ and $V_{e}=0$ or 1 . Now suppose that $\Lambda$ contains no bigon. Then each face has at least 3 sides and hence $3 F+V_{b} \leq 2 E$. Combining with $1=\chi$ (disk) $=V-E+F$, we get $E \leq 3 V-V_{b}-3=3 V_{i}+2 V_{b}-3$.

Since all boundary vertices but the exceptional vertex have valency at least 4 and all interior vertices have valency 6 , we have $4\left(V_{b}-V_{e}\right)+2 V_{e}+6 V_{i} \leq 2 E$. These two inequalities give $3 \leq V_{e}$, a contradiction. Hence $\Lambda$ contains a black bigon by Lemma 4.7 .

As in [13], we may label an edge $e$ of $G_{1}$ by class of the corresponding edge of $G_{2}$. We refer to this label as the edge class label of $e$. Then an edge of type 1 has edge class label $\alpha$ or $\beta$, and an edge of type 2 has edge class label $\gamma$ or $\delta$.

Let $M_{X}=M \cap X$ and $M_{Y}=M \cap Y$.

Lemma 4.9. Any two bigons in $G_{1}^{+}$have the same pair of edge class labels. Furthermore the pair is either $\{\alpha, \beta\}$ or $\{\gamma, \delta\}$.

Proof. If there are two bigons of $G_{1}^{+}$with distinct pairs of edge class labels, the argument in the proof of $\left[13\right.$, Lemma 5.2] shows that $M\left(r_{2}\right)$ contains a Klein bottle. This contradicts our assumption just above Lemma 4.4.

Now we shall show that the pair is either $\{\alpha, \beta\}$ or $\{\gamma, \delta\}$. By Lemma 4.6 there is a face $f$ of $\Lambda$ bounded only by edges of type 1, say. Thus the edges of $f$ lie in an essential annulus on $\widehat{F}_{2}$, so $f$ is black by Lemma 4.5 . Let $u_{x_{1}}, \ldots, u_{x_{n}}$ be the vertices of $f$ and let $\lambda_{i}$ be the corner of $f$ at $u_{x_{i}}$. As illustrated in in Figure 7 , let $I_{j}$ be the shortest interval on $\partial v_{j}$ containing the endpoints of edges of type 1 at $v_{j}$ for $j=1,2$.

As in the proof of Proposition 3.5, one can find a disk $D$ on the annulus $\partial H_{X}-\operatorname{Int}\left(v_{1} \cup v_{2}\right)$ such that $D \supset \lambda_{1} \cup \cdots \cup \lambda_{n}$ and $\partial D=a \cup b \cup c \cup d$, where $a$ and $c$ are respectively subarcs of $I_{1}$ and $I_{2}$, and $b$ and $d$ are essential arcs in the annulus $\partial H_{X}-\operatorname{Int}\left(v_{1} \cup v_{2}\right)$, parallel to each of $\lambda_{i}$. Let $A$ be the annulus in $F_{2}$ bounded by the edges $e_{1}$ and $e_{1}^{\prime}$ in Figure 7 along with subarcs on $\partial v_{1}$ and $\partial v_{2}$ containing $I_{1}$ and $I_{2}$. Then $A \cup D$ is a once punctured torus. Enlarging $D$ slightly in $\partial H_{X}-\operatorname{Int}\left(v_{1} \cup v_{2}\right)$ we may assume that $\partial f$ lies $\operatorname{in} \operatorname{Int}(A \cup D)$. Notice that $\partial f$ is a non-separating curve on $A \cup D$, since the vertices of $f$ are all parallel. Surgering $A \cup D$ along $f$ gives a disk $B$. Pushing $\operatorname{Int} B$ into the interior of $M_{X}$ rel. boundary gives a properly embedded disk $B^{\prime}$ in $M_{X}$. Here 
$\partial B^{\prime} \cap \partial H_{X}=b \cup d$ and $\partial B^{\prime}-\operatorname{Int}(b \cup d)$ consists of two arcs in $\partial A$. Notice that an orientation of $\partial B^{\prime}$ induces orientations of $b$ and $d$ which are opposite in the annulus $\partial H_{X}-\operatorname{Int}\left(v_{1} \cup v_{2}\right)$. Now by shrinking $H_{X}$ to its core, $H_{X} \cup B^{\prime}$ becomes a properly embedded annulus $A^{\prime}$ in $X$.

The annulus $A^{\prime}$ divides $X$ into two regions $Z_{1}$ and $Z_{2}$. We claim that both $Z_{1}$ and $Z_{2}$ are solid tori. Since the core of $H_{X}$ lies on $A^{\prime}$, we can isotope the core of $J_{2}$ slightly so that it is disjoint from the torus $\partial Z_{1}$. Then the minimality of $\left|\widehat{F}_{2} \cap J_{2}\right|$ guarantees that $Z_{1}$ is a solid torus. Similarly so is $Z_{2}$. Thus $X$ is a Seifert fibered space over the disk with the core of $A^{\prime}$ (and hence that of $A$ ) a Seifert fiber. Since $M\left(r_{2}\right)$ (and hence X) contains no Klein bottle, the Seifert fibration of $X$ is unique by [15, Theorem VI.18]. Therefore the pair of edge class labels of any bigon in $G_{1}^{+}$is either $\{\alpha, \beta\}$ or $\{\gamma, \delta\}$ by Lemma 4.7.

Lemma 4.10. (1) All interior vertices of $\Lambda$ have valency at least 4 in $\bar{\Lambda}$.

(2) All boundary vertices of $\Lambda$ but the exceptional vertex have valency at least 3 in $\bar{\Lambda}$.

Proof. (1) If $\Lambda$ had a set of three parallel edges, the set would contain a white bigon, contradicting Lemma 4.7. Since any interior vertex has valency 6 in $\Lambda$, it has valency at least 3 in $\bar{\Lambda}$. Suppose that an interior vertex has valency exactly 3 in $\bar{\Lambda}$. Then the vertex is incident to three pairs of parallel edges in $\Lambda$, which have the same pair of edge class labels, say, $\{\alpha, \beta\}$ by Lemma 4.9. This contradicts the property (1) of Proposition 4.3.

(2) Suppose that a non-exceptional boundary vertex of $\Lambda$ has valency 2 in $\bar{\Lambda}$. Then the vertex is incident to two pairs of parallel positive edges and two negative edges in $G_{1}$. Then we get a contradiction as above.

Lemma 4.11. $\Lambda$ contains a black trigon.

Proof. Let $V, E$ and $F$ be the number of vertices, edges and faces of $\bar{\Lambda}$, respectively. Let $V_{b}, V_{i}$ and $V_{e}$ denote the number of boundary vertices, interior vertices and the exceptional vertex of $\Lambda$, respectively. Now suppose that $\bar{\Lambda}$ contains no 3 -sided face. Then each face of $\bar{\Lambda}$ has at least 4 sides and hence $4 F+V_{b} \leq 2 E$. Combining $1=V-E+F$, we get $2 E \leq 4 V_{i}+3 V_{b}-4$.

By Lemma 4.10 we have $3\left(V_{b}-V_{e}\right)+2 V_{e}+4 V_{i} \leq 2 E$. These two inequalities give $4 \leq V_{e}$, a contradiction. Thus $\bar{\Lambda}$ (and hence $\Lambda$ ) has a 3 -sided face, which must be black by Lemma 4.7 .

Lemma 4.12. $M_{X}$ is a handlebody of genus 2 and $M_{Y}$ is a compression body with the boundary a union of a genus 2 surface and $\partial_{1} M$.

Proof. Since $F_{2}$ is a twice punctured torus, both $\partial M_{X}$ and $\partial M_{Y}-\partial_{1} M$ are surfaces of genus 2. By Lemma 4.10(2) one easily sees that $\Lambda$ contains black and white faces simultaneously. A black face compresses $\partial M_{X}$ to result in a torus in $M_{X}$, which bounds a solid torus since $M_{X}$ contains no incompressible torus. Hence $M_{X}$ is a handlebody of genus 2. Similarly a white face compresses $\partial M_{Y}-\partial_{1} M$ to result in a torus parallel to $\partial_{1} M$. 
Travelling around the boundary of a disk face of $G_{1}^{+}$gives rise to a cyclic sequence of edge class labels. We shall say that two disk faces of $G_{1}^{+}$of the same color are isomorphic if the cyclic sequences obtained by travelling in some directions are equal.

Let $A_{X}=H_{X} \cap M_{X}$ and $A_{Y}=H_{Y} \cap M_{Y}$. Then $\partial M_{X}=A_{X} \cup F_{2}$ and $\partial M_{Y}-\partial_{1} M=A_{Y} \cup F_{2}$. Since all the vertices of $\Lambda$ are parallel, each face of $\Lambda$ is a non-separating disk in $M_{X}$ or $M_{Y}$. Note that any two faces of $\Lambda$ of the same color are disjoint.

Lemma 4.13. If two disk faces of $G_{1}^{+}$are parallel in $M_{X}$ or $M_{Y}$, then they are isomorphic.

Proof. Suppose, for example, that two disk faces $f$ and $g$ of $G_{1}^{+}$are parallel in $M_{X}$. The curves $\partial f$ and $\partial g$ cobound an annulus $A$ in $\partial M_{X}$. Note that each component of $\partial A-\operatorname{Int} A_{X}$ is an edge of $G_{2}$ in $\partial f$ or $\partial g$, while each component of $\partial A \cap A_{X}$ is a corner of $f$ or $g$. The boundary circles of $A_{X}$ must intersect $A$ in spanning arcs, otherwise some edge of $G_{2}$ in $\partial f$ or $\partial g$ would be a trivial loop in $G_{2}$. Thus $A-\operatorname{Int} A_{X}$ is a union of disjoint rectangles $R_{1}, \ldots, R_{n}$. Each $R_{i}$ realizes a parallelism between two edges $\partial f \cap R_{i}$ and $\partial g \cap R_{i}$, so these edges have the same edge class label.

By Lemmas 4.8 and $4.11, G_{1}^{+}$contains black bigons and trigons. Without loss of generality we may assume that all bigons of $G_{1}^{+}$have the edge class label pair, $\{\alpha, \beta\}$, by Lemma 4.9. Then we have the following.

Lemma 4.14. All trigons of $G_{1}^{+}$have the same pair of edge class labels $\{\gamma, \delta\}$, i.e. they are bounded by edges of type 2 .

Proof. Let $f$ and $g$ be a bigon and a trigon of $G_{1}^{+}$, respectively. Let $A$ be the annulus in $F_{2}$ bounded by $e_{1}$ and $e_{1}^{\prime}$ along with two subarcs in $\partial F_{2}$ as shown in Figure 7. Then $f$ is bounded by an $\alpha$-edge and a $\beta$-edge, and $X$ is a Seifert fibered space over the disk with two exceptional fibers, whose Seifert fibration is unique because $X$ does not contain a Klein bottle. Here, the core of $A$ is a Seifert fiber.

By Lemma 4.7, $g$ is bounded either by edges of type 1 or by edges of type 2 . In the first case, surgering a twice punctured torus $A \cup A_{X}$ using $f$ and $g$ gives two disks in $X$, since $\partial f$ and $\partial g$ are non-separating and not mutually parallel in the surface. The boundary circles of the disks lie in $\widehat{F}_{2}$ and are isotopic to the core of $A$. This implies that $\widehat{F}_{2}$ is compressible in $M\left(r_{2}\right)$, a contradiction. Thus $g$ is bounded by edges of type 2 .

We will assume that each trigon has two $\gamma$-edges and a $\delta$-edge.

Lemma 4.15. If two edges $e_{1}, e_{2}$ of $G_{1}^{+}$are incident to a vertex with the same label, then they have distinct edge class labels.

Proof. If $e_{1}$ and $e_{2}$ have the same edge class labels, then the corresponding edge class in $G_{2}$ contains more than $n_{1}$ edges, contradicting Lemma 2.1(6). 
Lemma 4.16. There is no triple of mutually non-isomorphic black disk faces of $G_{1}^{+}$.

Proof. Suppose that $G_{1}^{+}$has such a triple $\left(f_{1}, f_{2}, f_{3}\right)$. Then these faces cut $M_{X}$ into two 3-balls by Lemma 4.13.

Claim. $G_{1}=G_{1}^{+}$and it is connected.

Proof. Note that $G_{1}$ is connected if and only if every face of $G_{1}$ is a disk. Let $f$ be a black face of $G_{1}$ other than $f_{1}, f_{2}, f_{3}$. Then $f$ lies in the complement of $f_{1} \cup f_{2} \cup f_{3}$ in $M_{X}$, so $f$ must be a disk, otherwise it would be compressible in $M_{X}$, so $F_{1}$ would be compressible in $M$ and one could find a new essential sphere in $M\left(r_{1}\right)$ which meets $J_{1}$ in fewer components than $\widehat{F}_{1}$. Each component of $\partial f \cap F_{2}$ is an edge of $G_{2}$. The circle $\partial f$ must be an essential curve in $\partial M_{X}=F_{2} \cup A_{X}$, otherwise some component of $\partial f \cap F_{2}$ would be a trivial loop in $G_{2}$. Hence $f$ is an essential disk in $M_{X}$, which must be parallel to one of the faces $f_{1}, f_{2}$ and $f_{3}$. Therefore any black face of $G_{1}$ is a disk face isomorphic to one of $f_{1}, f_{2}$ and $f_{3}$ by Lemma 4.13. It follows that all the edges of $G_{1}$ are positive, i.e. $G_{1}=G_{1}^{+}$.

It remains to show that any white face of $G_{1}$ is a disk. Suppose to the contrary that a white face $g$ of $G_{1}$ is not a disk. Then $g$ is incompressible in $M_{Y}$ as above. Recall that $\Lambda$ contains a white disk face and that $M_{Y}$ is a compression body with the outer boundary a surface of genus 2 and the inner boundary a torus, $\partial_{1} M$. Let $g_{1}$ be a white disk face of $\Lambda$. Cutting $M_{Y}$ along $g_{1}$ gives rise to a manifold $V$, homeomorphic to $S^{1} \times S^{1} \times I$, with $\partial_{1} M$ one component of $\partial V$. Here, $g$ lies in $V$ and $\partial g \subset \partial V-\partial_{1} M$. Since $g$ is incompressible in $M_{Y}, g$ is also incompressible in $V$. Hence $g$ must be an annulus parallel to $\partial V-\partial_{1} M$. Thus $V$ contains an annulus $A$ such that one component of $\partial A$ is the core curve of $g$ and the other lies in $\partial_{1} M$, where $\partial A \cap \partial_{1} M$ is an essential curve, otherwise $g$ would be compressible in $M_{Y}$. Surgering $\widehat{F}_{1}$ along $A$ gives two compressing disks for $\partial_{1} M$ in $M\left(r_{1}\right)$. This shows $M\left(r_{1}\right)$ is boundary-reducible. The assumption $\Delta=3$ contradicts [13, Theorem 1.1].

Hence, $\widehat{F}_{1}$ is a non-separating sphere in $M\left(r_{1}\right)$. This contradicts [16, Theorem $1.1]$.

Proof of Theorem 1.1. Orient the edges of $G_{1}$ as shown in Figure 8 so that $G_{1}$ becomes a directed graph in the 2 -sphere $\widehat{F}_{1}$. (For example, all $\alpha$-edges are oriented so that their left hand sides are black.)

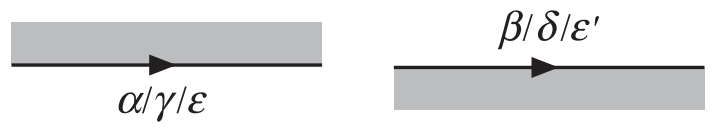

Figure 8

Note that any disk face of $G_{1}$ has the same number of $\varepsilon$-edges and $\varepsilon^{\prime}$-edges. Hence if there were a cycle face in $G_{1}$, then it would be a face of $G_{1}^{+}$, since the 
$\varepsilon$-edges and $\varepsilon^{\prime}$-edges are oppositely oriented. Moreover, it would be bounded either by $\alpha$-edges and $\gamma$-edges or by $\beta$-edges and $\delta$-edges and hence it would be white by Lemma 4.16. This contradicts Lemma 4.5.

Therefore it is enough to show that $G_{1}$ has neither a sink nor a source. Assume for contradiction that $G_{1}$ has a source at a vertex $u_{x}$. The local view at $u_{x}$ must be like one of the pictures in Figure 9 by Lemma 4.15. We shall show that any of them is impossible.

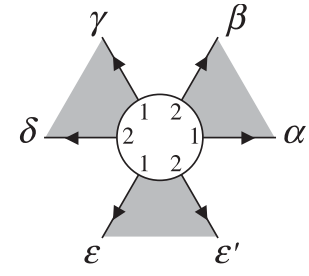

(a)

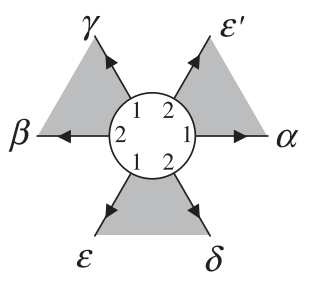

(e)

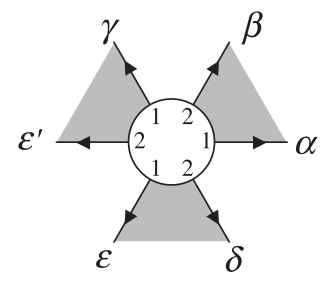

(i)

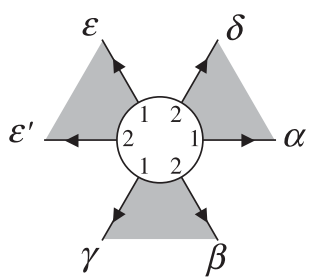

(b)

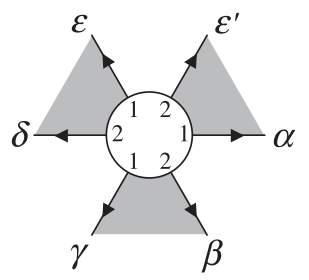

(f)

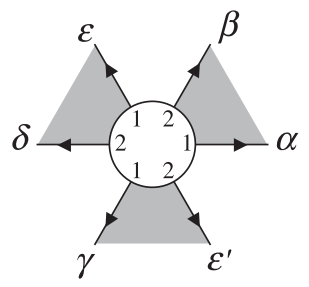

(j)

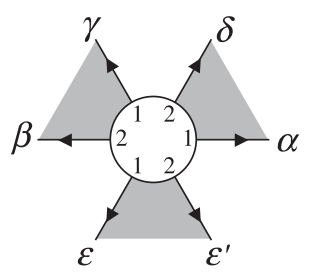

(c)

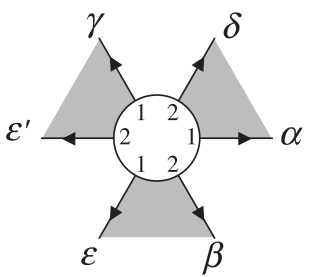

$(\mathrm{g})$

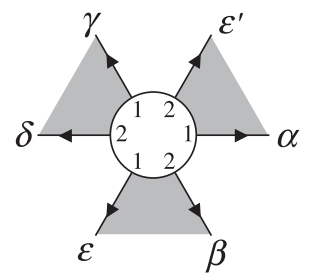

(k)

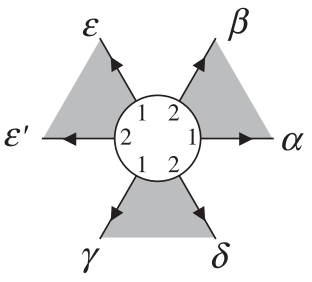

(d)

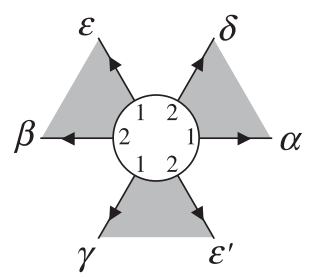

(h)

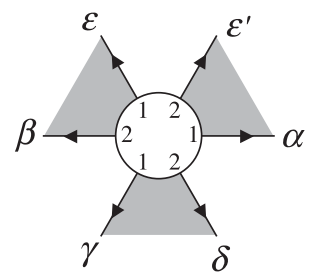

(1)

FiguRE 9

In $G_{2}$, the label $x$ appears three times around the vertex $v_{1}$ at ends of $\alpha_{-}, \gamma_{-}$, and $\varepsilon$-edge families and three times around the vertex $v_{2}$ at ends of $\beta-, \delta$-, and $\varepsilon^{\prime}$-edge families.

Claim. In $G_{2}$, the label $x$ appears at the northern (resp. southern) end of $\varepsilon$-edge family if and only if it appears at the southern (resp. northern) end of $\varepsilon^{\prime}$-edge family.

Proof. Assume, for example, that $x$ appears at the northern ends of $\varepsilon$ - and $\varepsilon^{\prime}$-edge families. See Figure 10. Around the vertex $v_{1}, x$ does not appear at an end of $\delta$-edge family and at the southern end of $\varepsilon$-edge family, implying $w(\delta)+w(\varepsilon)<n_{1}$. Around the vertex $v_{2}, x$ appears once at an end of $\delta$-edge 
family and once at the northern end of $\varepsilon^{\prime}$-edge family, implying $w(\delta)+w\left(\varepsilon^{\prime}\right)>n_{1}$. Since $w(\varepsilon)=w\left(\varepsilon^{\prime}\right)$, these two inequalities conflict.

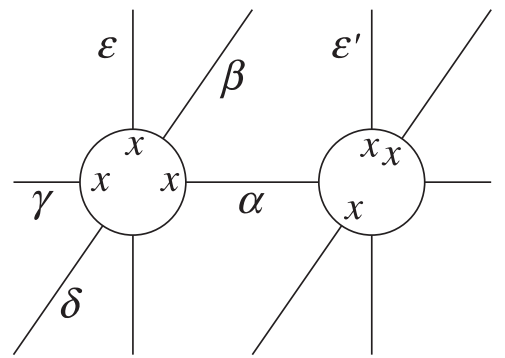

FigURE 10

Let $f$ be a black bigon of $\Lambda$ with vertices $u_{y}, u_{z}$ and $g$ a black trigon with vertices $u_{p}, u_{q}, u_{r}$, as shown in Figure 11. Let $C_{1}$ be the corner of $f$ at the vertex $u_{z}$ and $C_{2}$ the corner of $g$ at the vertex $u_{q}$.
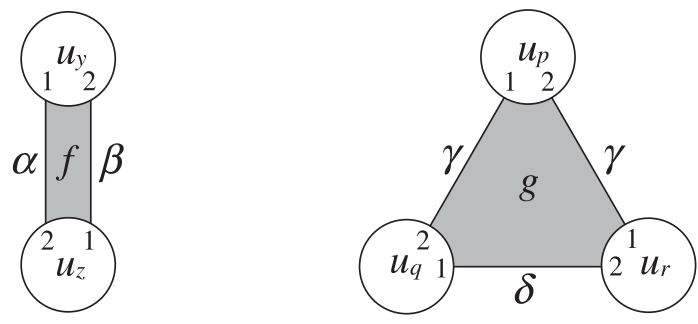

\section{FigURE 11}

Assume the source at $u_{x}$ looks like Figure 9(a), (b), (c) or (d). Let $C$ be the black corner in $\partial u_{x}$ running from an $\varepsilon$-edge endpoint to an $\varepsilon^{\prime}$-edge endpoint. Then $C, C_{1}, C_{2}$ contradict [14, Lemma 3.2] by the above claim.

Assume the source at $u_{x}$ looks like Figure $9(\mathrm{e})$ or (f). Let $C$ be the black corner in $\partial u_{x}$ running from a $\gamma$-edge endpoint to a $\beta$-edge endpoint. Then $C, C_{1}, C_{2}$ contradict [14, Lemma 3.2].

Assume the source at $u_{x}$ looks like Figure $9(\mathrm{~g})$ or $(\mathrm{h})$. Let $C$ be the black corner in $\partial u_{x}$ running from an $\alpha$-edge endpoint to a $\delta$-edge endpoint. Then $C, C_{1}, C_{2}$ contradict [14, Lemma 3.2].

Assume the source at $u_{x}$ looks like Figure $9(\mathrm{i})$ or $(\mathrm{j})$. Let $C$ be the black corner in $\partial u_{x}$ running from a $\gamma$-edge endpoint to an $\varepsilon^{\prime}$-edge endpoint and $C^{\prime}$ the black corner running from an $\varepsilon$-edge endpoint to a $\delta$-edge endpoint. Then $C, C^{\prime}, C_{2}$ contradict $[14$, Lemma 3.2$]$ by the above claim.

Assume the source at $u_{x}$ looks like Figure $9(\mathrm{k})$ or $(\mathrm{l})$. Let $C$ be the black corner in $\partial u_{x}$ running from an $\varepsilon$-edge endpoint to a $\beta$-edge endpoint and $C^{\prime}$ the black corner running from an $\alpha$-edge endpoint to an $\varepsilon^{\prime}$-edge endpoint. Then $C, C^{\prime}, C_{1}$ contradict $[14$, Lemma 3.2$]$ by the above claim.

Using the same argument as above, we can see that $G_{1}$ has no sink. 
I would like to thank the referee and Sungmo Kang for pointing out errors in the original texts and helpful comments.

\section{References}

[1] S. Boyer and X. Zhang, Reducing Dehn filling and toroidal Dehn filling, Topology Appl. 68 (1996) 285-303.

[2] _ Finite Dehn surgery on knots, J. Amer. Math. Soc. 9 (1996) 1005-1050.

[3] _ On Culler-Shalen seminorms and Dehn filling, Ann. of Math. (2) 148 (1998) $737-801$.

[4] M. Culler, C. McA. Gordon, J. Luecke, and P.B. Shalen, Dehn surgery on knots, Ann. of Math. (2) 125 (1987), 237-300.

[5] M. Eudave-Muñoz and Y. Q. Wu, Nonhyperbolic Dehn fillings on hyperbolic 3-manifolds, Pacific J.Math. 190 (1999), 261-275.

[6] C. McA. Gordon, Boundary slopes on punctured tori in 3-manifolds, Trans. Amer. Math. Soc. 350 (1998), 1713-1790.

[7] _ Dehn filling: a survey, Knot theory (Warsaw, 1995), 129-144, Banach Center Publ., 42, Polish Acad. Sci., Warsaw, 1998.

[8] _ Small surfaces and Dehn fillngs, Geom. and Topol. Mono. 2 (1999), 177-199.

[9] C. McA. Gordon and R. Litherland, Incompressible planar surfaces in 3-manifolds, Topology Appl. 18 (1984), 121-144.

[10] C. McA. Gordon and J. Luecke, Knots are determined by their complements, J. Amer. Math. Soc. 2 (1989), 371-415.

[11] _ Dehn surgeries on knots creating essential tori, I, Comm. Anal. and Geom. 3 (1995), 597-644.

[12] _ Reducible manifolds and Dehn surgery, Topology 35 (1996), 385-409.

[13] _ Toroidal and boundary-reducing Dehn fillings, Topology Appl. 93 (1999), 77-90.

[14] _ Non-integral toroidal Dehn surgeries, Comm. Anal. and Geom. 12 (2004) 417485.

[15] W. Jaco, Lectures on three-manifold topology, Regional Conf. Series in Math. 43 (1997).

[16] S. Lee, Toroidal Dehn surgeries on knots in $S^{1} \times S^{2}$, J. Knot Theory Ramifications 14 (2005), 657-664.

[17] S. Lee, S. Oh and M. Teragaito, Reducing Dehn fillings and small surfaces, to apper in Proc. London Math. Soc.

[18] S. Oh, Reducible and toroidal manifolds obtained by Dehn fillings, Topology Appl. 75 (1997), 93-104.

[19] , Dehn filling, reducible 3-manifolds and Klein bottles, Proc. Amer. Math. Soc. 126 (1998), 289-296.

[20] M. Scharlemann, Producing reducible 3-manifolds by surgery on a knot, Topology 29 (1990), 481-500.

[21] Y.Q. Wu, The reducibility of surgered 3-manifolds, Topology Appl. 43 (1992), 213-218.

$[22]$, Sutured manifold hierarchies, essential laminations, and Dehn surgery, J. Diff. Geom. 48 (1998), 407-437.

[23] _ Dehn fillings producing reducible manifolds and toroidal manifolds, Topology $\mathbf{3 7}$ (1998), 95-108.

School of Mathematics, Korea Institute for Advanced Study 207-43 Cheongryangri-dong, Dongdaemun-gu Seoul 130-722, Korea

E-mail address: slee@kias.re.kr 